\title{
1. Molecular characteristics and antimicrobial 4 resistance of group B streptococcus strains causing invasive disease in neonates and adults
}

Jen-Fu Hsu, MD; Ming-Horng Tsai, MD ${ }^{1,4}$; Mei-Yin Lai, MD ${ }^{2,4}$; Lee-Chung Lin, PhD ${ }^{3}$; Shih-Ming Chu, MD ${ }^{2,4}$; Hsuan-Rong Huang, $\mathrm{MD}^{2,4}$; Ming-Chou Chiang, MD ${ }^{2,4}$; Ren-Huei Fu, MD PhD ${ }^{2,4}$; and Jang-Jih Lu, MD, $\mathrm{PhD}^{3,4,5^{*}}$

We aimed to analyze the molecular characteristics, clonality and antimicrobial resistance profiles of group B streptococcus (GBS) isolates collected in Taiwan from invasive diseases and carriage. Multilocus sequence typing (MLST) was used to assess the genetic diversity of 225 GBS strains from neonates and adults with invasive GBS diseases. 100 GBS strains collected from colonized pregnant women during the same period were compared, and all strains were characterized for one of nine capsule genotypes. We also determined the susceptibilities of all GBS isolates to various antimicrobial agents. The most frequently identified serotypes that caused invasive disease in neonates were III $(60.6 \%)$ and Ia $(17.3 \%)$, whereas type VI $(32.7 \%)$, Ib $(19.4 \%)$, and $\mathrm{V}(19.4 \%)$ were the most common to cause invasive disease in adults. Serotype VI was the leading type that colonized pregnant women (35.0\%). Twenty-six sequence types (STs) were identified, and $90.5 \%$ of GBS strains were represented by 6 STs. ST-17 and ST-1 were more prevalent in invasive diseases in neonates and adults, respectively. ST-17 strains were more likely to cause meningitis and late-onset disease than other strains. In addition, ST-12 and ST-17 GBS strains showed the highest rate of resistance to erythromycin and clindamycin (range: 75.8-100\%). In conclusion, CC-17/type III and CC$1 /$ type VI are the most important invasive pathogens in infants and nonpregnant adults in Taiwan, respectively. GBS genotypes vary between different age groups and geographical areas and should be considered during GBS vaccine development.

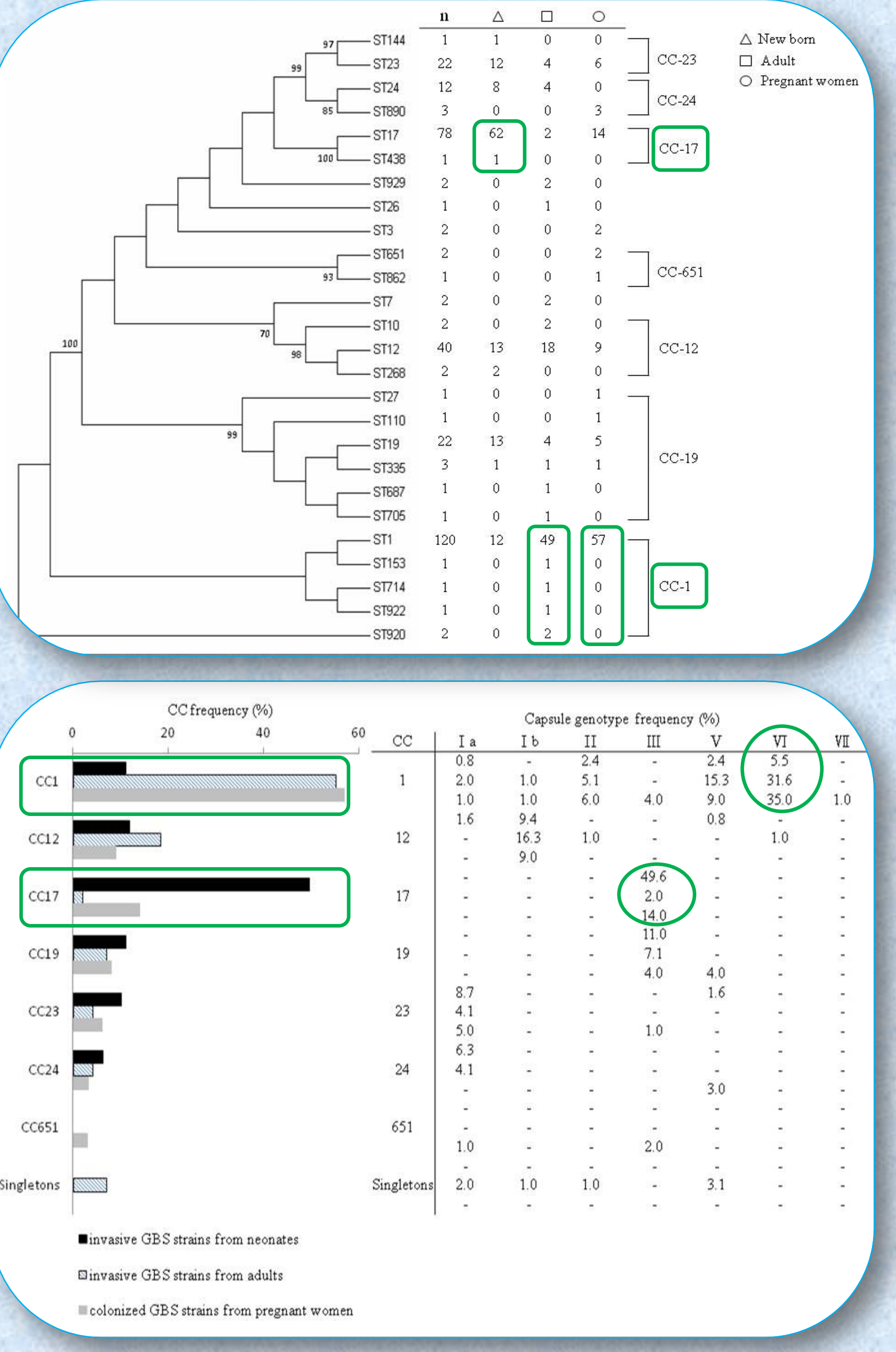

Purpose: We aimed to analyze the molecular characteristics, clonality and antimicrobial resistance profiles of GBS isolates collected in Taiwan from invasive diseases and carriage.

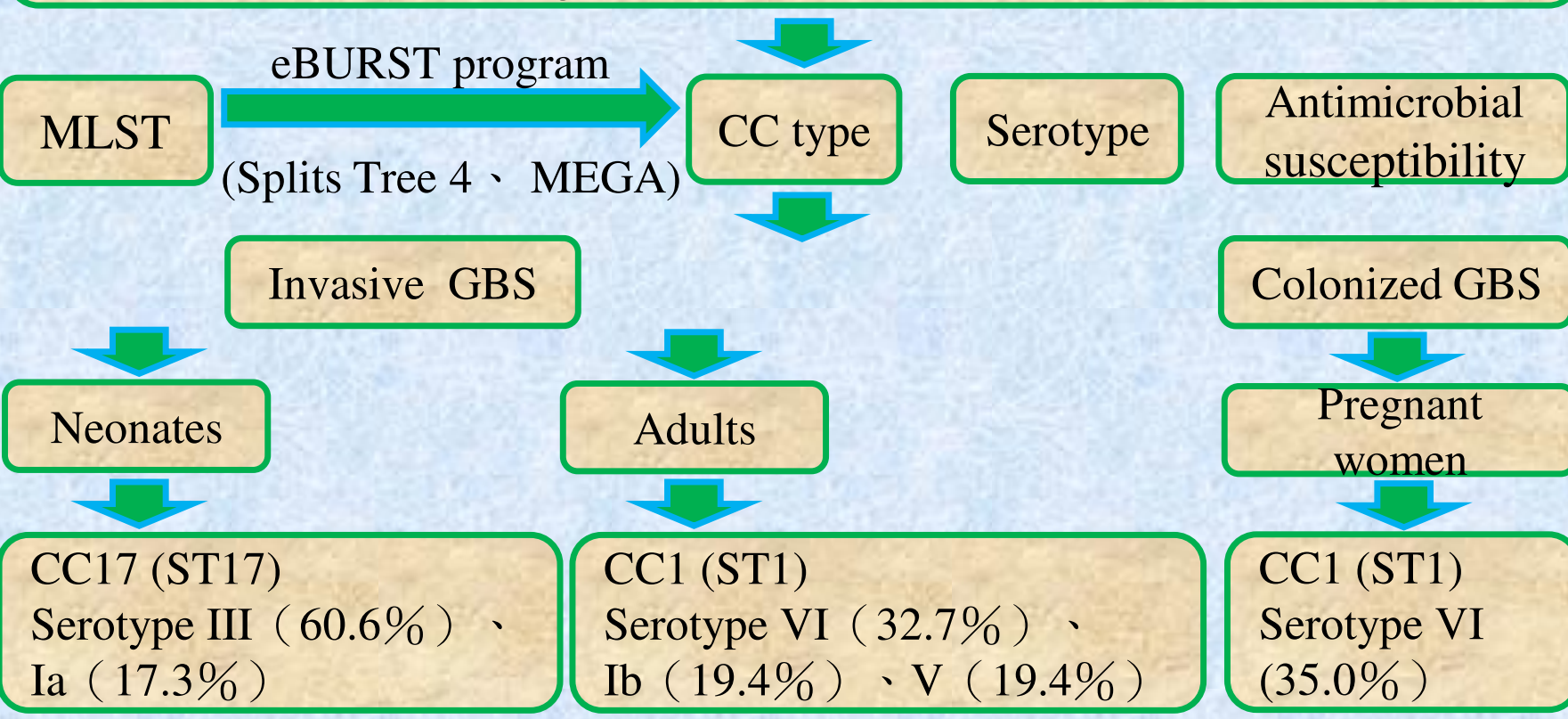

ST-17 strains were more likely to cause meningitis and late-onset disease than other strains

ST-12 and ST-17 GBS strains showed the highest rate of resistance to erythromycin and clindamycin (range: $75.8-100 \%$ )

\section{$+1$}

Conclusion : CC-17/type III and CC-1/type VI are the most important invasive pathogens in infants and adults in Taiwan, respectively. And GBS genotypes vary between different age groups and geographical areas and should be considered during GBS vaccine development.

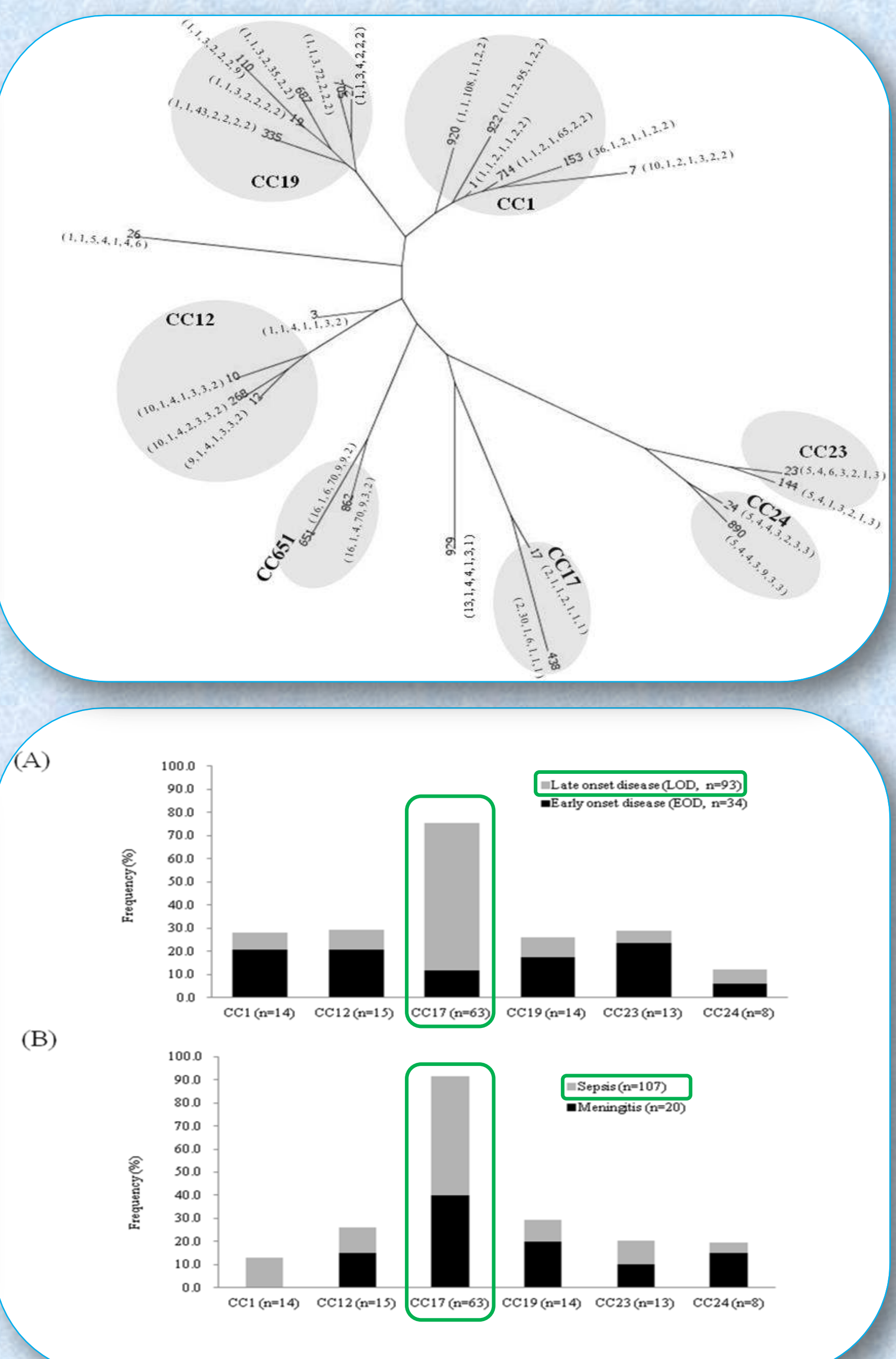

